Article

\title{
Exact and Slow-Roll Solutions for Exponential Power-Law Inflation Connected with Modified Gravity and Observational Constraints
}

\author{
Igor Fomin ${ }^{1, *(D)}$ and Sergey Chervon ${ }^{1,2,3}$ (D) \\ 1 Department of Physics, Bauman Moscow State Technical University, 2-nd Baumanskaya Street, 5, \\ 105005 Moscow, Russia; chervon.sergey@gmail.com \\ 2 Department of Physics and Technical Discipline, Ulyanovsk State Pedagogical University, Lenin's Square, \\ 4/5, 432071 Ulyanovsk, Russia \\ 3 Institute of Physics, Kazan Federal University, Kremlevskaya Street 18, 420008 Kazan, Russia \\ * Correspondence: ingvor@inbox.ru
}

Received: 14 September 2020; Accepted: 26 October 2020; Published: 29 October 2020

check for updates

\begin{abstract}
We investigate the ability of the exponential power-law inflation to be a phenomenologically correct model of the early universe. We study General Relativity (GR) scalar cosmology equations in Ivanov-Salopek-Bond (or Hamilton-Jacobi like) representation where the Hubble parameter $H$ is the function of a scalar field $\phi$. Such approach admits calculation of the potential for given $H(\phi)$ and consequently reconstruction of $f(R)$ gravity in parametric form. By this manner the Starobinsky potential and non-minimal Higgs potential (and consequently the corresponding $f(R)$ gravity) were reconstructed using constraints on the model's parameters. We also consider methods for generalising the obtained solutions to the case of chiral cosmological models and scalar-tensor gravity. Models based on the quadratic relationship between the Hubble parameter and the function of the non-minimal interaction of the scalar field and curvature are also considered. Comparison to observation (PLANCK 2018) data shows that all models under consideration give correct values for the scalar spectral index and tensor-to-scalar ratio under a wide range of exponential-power-law model's parameters.
\end{abstract}

Keywords: gravitation; cosmology; inflation

\section{Introduction}

The inflationary paradigm implying the accelerated expansion of the early universe is often considered as a successful [1] explanation for the origin of its structure. The first models of cosmological inflation were built on the basis of General Relativity (GR) in 4D Friedmann-Robertson-Walker (FRW) space-time under the assumption of the existence of some scalar field (inflaton), which is the source of the accelerated expansion of the universe [1-4].

Since the potential of a scalar field $V(\phi)$ has a key role in the construction of inflation scenarios, the models of the early universe are often determined by the chosen potential. Such a method can be called as the "potential motivated approach". In this case, inflationary models are classified by the scalar field potential.

On the other hand, the dynamics of the expansion, which are characterised by a scale factor $a(t)$, are no less important for an understanding of the inflationary scenarios and one can reconstruct the potential $V(\phi)$ from the chosen scale factor (see, for example, [5]). In the context of this method, which we will call the "dynamical motivated approach", inflationary models are classified by the laws of the expansion of the universe. 
A convenient tool for the analysis of the inflationary model is the slow-roll approximation (see, for example, in [6]) and a lot of models are considered on the basis of this approach. We also note the possibility of constructing quasi de Sitter solutions based on the linear relationship between the kinetic energy of a scalar field and the state parameter in the context of kinetic approximation $[7,8]$. Nevertheless, for a more correct understanding of the nature of processes at the stage of inflation, the exact solutions of cosmological dynamic equations were considered as well (see, for example, [9-14]). The classification of the methods for generating them (and the exact solutions themselves) for inflation based on Einstein gravity can be found in the monograph [15].

The accelerated expansion of the universe, discovered in 1998 by two independent collaborations $[16,17]$, significantly changed the paradigm of constructing cosmological models. To explain inflationary and second accelerated expansion, various types of scalar fields and interactions between them (in multifield models) or modifications and Einstein gravity that appear on cosmological scales are used.

According to modern observations, the dynamics of the universe are in good agreement with the $\Lambda \mathrm{CDM}$ model, which includes the cosmological constant associated with the vacuum energy [18-21] and simulates dark energy. An alternative description of dark energy is based on light fields of quintessence and $k$-essence, which are used instead of the cosmological constant [22-28]. As another source of the accelerated expansion of the universe [29-34], nonlinear sigma models with an interaction potential or chiral cosmological models with a multiplet of scalar fields were considered, the specificity of which is in describing the interaction between fields through the internal targets space. In the case of the description of the repeated accelerated expansion in the universe with cold dark matter, such models are called $\sigma$ CDM-models [31-34].

When studying cosmological models based on modified theories of gravity the question arises about the need to use scalar fields to explain the accelerated expansion of the universe. An example of a theory of gravity that successfully explains both stages of accelerated expansion and leads to a geometric interpretation of the cosmological constant [35] is $f(R)$-gravity [36-38], in which the dynamic effects of scalar fields (without including the scalar fields themselves) are obtained by adding higher-order curvature terms to the action.

Nevertheless, the equations of cosmological dynamics in $f(R)$-gravity can be considered in the framework of conformal connection with standard inflation models based on general relativity, containing the scalar field $[39,40]$. Based on this connection, the potential of the scalar field $V(\phi)$ can be reconstructed with the subsequent analysis of the cosmological model obtained in the Einstein representation [41]. On the other hand, it is possible to solve the inverse problem of reconstructing the dependence $f=f(R)$ from the known potential of the scalar field $V(\phi)$, that is, to obtain the form of $f(R)$-gravity, corresponding to the models considered in the Einstein representation cosmological inflation.

Another example of the early universe models based on the modifications of Einstein gravity is cosmological inflation based on scalar-tensor theories of gravity. The main attention is paid to the analysis of these models in this study. In [42-44], cosmological models based on tensor-scalar gravity were considered both for constructing actual models of the universe and for solving the inverse problem of reconstructing the parameters of the theory of gravity from the observed dynamics.

The most general scalar-tensor theory of gravity, leading to the equations of second-order cosmological dynamics in the four-dimensional Friedmann universe, is Horndeski gravity [45-47]. When constructing cosmological models based on Horndeski gravity, one can consider special cases based on the non-minimal interaction of the scalar field with the Ricci scalar [48-52] and the Gauss-Bonnet scalar [53-62], otherwise the Einstein-Gauss-Bonnet (EGB) gravity, which is also a special case of Lovelock gravity [63].

The inflationary models containing a combination of Friedmann solutions and (quasi) de Sitter solutions constitute the basis of an actual description of the evolution of the early universe. In the context of the inflationary paradigm, the early universe expands rapidly for some time and then goes 
into a power-law expansion regime without acceleration corresponding to the Friedmann solutions for radiation and baryonic matter.

In this article we consider the exponential power-law (EPL) inflation, which implies such a combination of the dynamical regimes. We also give an review of methods for constructing exact and approximate solutions with these types of dynamics for various types of gravity theories. For the case of $f(R)$-gravity on the basis of the exact solutions and ones obtained in slow-roll approximation it is shown that widely discussed Starobinsky [1,39,40,64-66] and non-minimal Higgs [67-69] inflationary models, which imply the same potential of the scalar field, can be considered as the partial cases of the exponential power-law inflation in the context of the dynamical motivated approach. We also generalize the cosmological solutions for EPL inflation obtained in the framework of general relativity and one scalar field to the cases of multifield chiral cosmological models and modified gravity theories with non-minimal coupling of the scalar field with the Ricci scalar and Gauss-Bonnet scalar. Further, we will consider EPL inflationary models with scalar-tensor gravity based on the quadratic relationship between the Hubble parameter and the function of the non-minimal coupling of the scalar field and curvature.

The article is organised as follows. Section 2 contains exact and slow-roll GR cosmology equations in FRW space-time. In Section 3 we analyse the slow-roll solutions for exponential power-law (EPL) inflation. Three parametric slow-roll approximated solutions were found and it is shown which restrictions on parameters lead to Starobinsky potential (which, in turn, leads to Starobinsky $R+R^{2}$ gravity model). The derivation of the de Sitter solution, and Starobinsky gravity using special choice of EPL model parameters are shown. The relation between exact and slow-roll solutions is discussed as well. In Section 4 we generalize the solutions obtained for EPL inflation in the framework of GR on the case of Chiral Cosmological Models (CCM) and describe the method of reducing cosmological solutions for single field models to multifield ones. In Section 5 we consider the GR-like cosmological models with EPL dynamics based on the scalar-tensor gravity implying non-minimal coupling of a scalar field with the Ricci scalar and Gauss-Bonnet scalar. In Section 6 correspondence EPL inflation to observation data was considered. It was shown that one of the parameters can always be chosen so that observational constraints are satisfied. Section 7 is devoted to EPL inflationary models based on the scalar-tensor gravity with quadratic connection between Hubble parameter and coupling function. Methods for constructing exact and approximate cosmological solutions for these models are also presented. In Section 8 we discuss the main results and methods under consideration.

\section{The Exact and Approximate Solutions for EPL Inflation Based on Einstein Gravity}

The inflationary models based on Einstein gravity and a single scalar field defined by the action

$$
S=\int d^{4} x \sqrt{-g}\left[\frac{1}{2} R-\frac{1}{2} g^{\mu v} \partial_{\mu} \phi \partial_{\nu} \phi-V(\phi)\right],
$$

where $\phi$ is a scalar field, $V(\phi)$ is the potential of a scalar field and $g^{\mu v}$ is a metric tensor of a space-time. We set Einstein gravitational constant $\kappa=8 \pi G=1$.

The variation of the action (1) with respect to the metric and field in a spatially flat Friedmann-Robertson-Walker space

$$
d s^{2}=-d t^{2}+a^{2}(t)\left(d x^{2}+d y^{2}+d z^{2}\right)
$$

gives three dynamic equations

$$
\begin{gathered}
3 H^{2}=\frac{1}{2} \dot{\phi}^{2}+V(\phi) \equiv \rho_{\phi}, \\
-3 H^{2}-2 \dot{H}=\frac{1}{2} \dot{\phi}^{2}-V(\phi) \equiv p_{\phi}, \\
\ddot{\phi}+3 H \dot{\phi}+V_{\phi}^{\prime}=0,
\end{gathered}
$$


where $\rho_{\phi}$ and $p_{\phi}$ are the energy density and the pressure of a scalar field, also, $V_{\phi}^{\prime}=d V / d \phi$.

The methods of the exact solutions construction for these systems of equations one can find, for example, in [11,12]. One of them is the method proposed by Ivanov [9] and subsequently Salopek and Bond [10].

From the Equations (3)-(5) only two are independent, and this system can be represented as the Ivanov-Salopek-Bond equations (or as Hamilton-Jacobi type equations)

$$
\begin{aligned}
& V(\phi)=3 H^{2}-2 H_{\phi}^{\prime 2}, \\
& \dot{\phi}=-2 H_{\phi}^{\prime}
\end{aligned}
$$

in which the exact solutions are obtained by the choice of the Hubble parameter $H(\phi)$.

In the case of the slow-roll approximation, which implies that $V(\phi) \gg \frac{1}{2} \dot{\phi}^{2}$ and $\ddot{\phi} \approx 0$, the system (3)-(5) is reduced to the equations

$$
\begin{aligned}
& V(\phi) \approx 3 H^{2}, \\
& \dot{\phi} \approx-2 H_{\phi}^{\prime} .
\end{aligned}
$$

Therefore, the difference between the exact and approximate background cosmological solutions is the second term in the potential (6).

\subsection{The Exact and Approximate Solutions for Exponential Power-Law Inflation}

We represent the exponential power-law (EPL) expansion of universe, with the following parametrisation of the Hubble function

$$
H(\phi)= \pm \mu_{1} \exp \left(-\mu_{2} \phi\right)+\mu_{3}
$$

where $\mu_{1}, \mu_{2}$ and $\mu_{3}$ are arbitrary real constants.

From Equations (6) and (7) one has the following exact solutions:

$$
\begin{aligned}
& V(\phi)=3\left(\mu_{3} \pm \mu_{1} e^{-\mu_{2} \phi}\right)^{2}-2 \mu_{1}^{2} \mu_{2}^{2} e^{-2 \mu_{2} \phi}, \\
& \phi(t)=\frac{1}{\mu_{2}} \ln \left(2 \mu_{1} \mu_{2}^{2} t+c\right)+n \frac{i \pi}{\mu_{2}}, \\
& H(t)=\frac{\mu_{1}}{2 \mu_{1} \mu_{2}^{2} t+c}+\mu_{3}, \\
& a(t)=a_{0} \exp \left(\mu_{3} t\right)\left(2 \mu_{1} \mu_{2}^{2} t+c\right)^{1 / 2 \mu_{2}^{2}}
\end{aligned}
$$

where the constant $n=0, \pm 1, \pm 2, \pm 3, \ldots$

For the case of the slow-roll approximation, from Equations (8)-(9) one has

$$
V_{S R}(\phi)=3\left(\mu_{3} \pm \mu_{1} e^{-\mu_{2} \phi}\right)^{2},
$$

with the same evolution of the scalar field and expansion law of the universe, which are defined by expressions (12)-(14).

As one can see, the scalar field (12) can be considered as a complex one with the variable real part and constant imaginary part.

Taking into account the Euler identity $e^{i \pi}=-1$, after substituting the expression (12) into (10) for even values of the constant $n$ one has potential (11) with an upper sign, and for odd values of the constant $n$ one has potential (11) with a lower sign corresponding to the same dynamics of the universe's expansion (13) and (14). For the constant $n=0$ one has potential (11) with upper sign corresponding to the case of the exponential power-law inflation with the canonical scalar field. 
Also, we note, that the parameters of the EPL inflationary model corresponding to the scalar field (12), namely kinetic energy, potential, energy density and pressure of this field

$$
\begin{aligned}
& X(t)=\frac{1}{2} \dot{\phi}^{2}=\frac{2 \mu_{1}^{2} \mu_{2}^{2}}{\left(2 \mu_{1} \mu_{2}^{2} t+c\right)^{2},} \\
& V(t)=\frac{\mu_{1}^{2}\left(3-2 \mu_{2}^{2}\right)}{\left(2 \mu_{1} \mu_{2}^{2} t+c\right)^{2}}+\frac{6 \mu_{1} \mu_{3}}{2 \mu_{1} \mu_{2}^{2} t+c}+3 \mu_{3}^{2}, \\
& \rho_{\phi}(t)=3\left(\frac{\mu_{1}}{2 \mu_{1} \mu_{2}^{2} t+c}+\mu_{3}\right)^{2}, \\
& p_{\phi}(t)=\frac{\mu_{1}^{2}\left(4 \mu_{2}^{2}-3\right)}{\left(2 \mu_{1} \mu_{2}^{2} t+c\right)^{2}}-\frac{6 \mu_{1} \mu_{3}}{2 \mu_{1} \mu_{2}^{2} t+c}-3 \mu_{3}^{2},
\end{aligned}
$$

are the real functions of cosmic time.

\subsection{The Dynamics of the Universe's Expansion}

Requesting a positive sign for $e^{\mu_{2} \phi}$ (or, equivalently, positive value for logarithm's argument of (12)) we have the restriction on time

$$
t<t_{\text {end }}, t_{\text {end }}=c /\left(2 \mu_{1} \mu_{2}^{2}\right),
$$

when $c \mu_{1}<0$. It means that till that time early inflation should finish.

Further restriction we obtain from universe expansion, i.e., $H>0$.

From Equation (13) we can find that $H$ will be positive always if

$$
\mu_{3}>0, \mu_{1}<0, c>0 \text {. }
$$

If $\mu_{3}<0$ the first term in the RHS of (13) should be positive and excess $\left|\mu_{3}\right|$. This leads to restriction on time

$$
t<\left|\frac{\mu_{1} /\left|\mu_{3}\right|-c}{2 \mu_{1} \mu_{2}^{2}}\right|,
$$

which is valid for $c>0, \mu_{1}<0$ or $c<0, \mu_{1}>0$. So the universe will be under expansion till the time (22), which should be less than that validated by the solution, the time $t_{\text {end }}(20)$. Therefore the inequality

$$
\left|\mu_{3}\right|<\mu_{1} /(2 c)
$$

should be true.

Let us study the period of acceleration for the model under consideration under the condition (20). Direct calculation of relative acceleration

$$
Q \equiv \ddot{a} / a=H^{2}+\dot{H},
$$

gives

$$
Q=\frac{\ddot{a}}{a}=\mu_{1}^{2} e^{-2 \mu_{1} \phi}\left(1-2 \mu_{2}^{3}\right)-2 \mu_{1} \mu_{3} e^{-\mu_{2} \phi}+\mu_{3} .
$$

Generally speaking the case when $\left(1-2 \mu_{2}^{3}\right)>0$ gives two accelerating periods in terms of $e^{-\mu_{2} \phi}$ when $\mu_{3}<0$ and when $0<\mu_{3}<1 / \sqrt{2}$. If $\left(1-2 \mu_{2}^{3}\right)<0$ then there exists one accelerating period in the cases: (i) $\mu_{3}>1 / \sqrt{2}$ solution oscillates between two roots; (ii) $0<\mu_{3}<1 / \sqrt{2}$ solution oscillates between zero and the bigger root; (iii) the same behaviour when $\mu_{3}<-1 / \sqrt{2}$.

Also, we note that a numerical analysis of the stages of expansion of the universe for the Hubble parameter (13) was performed in [70]. 
We also give an asymptotic analysis of the dynamics of expansion of the universe for the case of positive values of the constant parameters $\mu_{1}, \mu_{3}$ and $c$.

At the small times that correspond to inflationary stage $t \approx 0$ one has the exponential expansion with following Hubble parameter and scale factor

$$
H_{i n f} \simeq \frac{\mu_{1}}{c}+\mu_{3}, \quad a_{i n f}(t) \propto \exp \left[\left(\frac{\mu_{1}}{c}+\mu_{3}\right) t\right]
$$

On the following stage, under the condition

$$
\frac{\mu_{1}}{2 \mu_{1} \mu_{2}^{2} t+c} \gg \mu_{3}
$$

one has the power-law expansion with the Hubble parameter and scale factor

$$
H_{P L}(t) \simeq \frac{\mu_{1}}{2 \mu_{1} \mu_{2}^{2} t+c}, \quad a_{P L}(t) \propto\left(2 \mu_{1} \mu_{2}^{2} t+c\right)^{1 / 2 \mu_{2}^{2}} .
$$

At the large times $t \rightarrow \infty$ we have the second accelerated exponential expansion of the universe with

$$
H_{s e c} \simeq \mu_{3}, \quad a_{s e c}(t) \propto \exp \left(\mu_{3} t\right) .
$$

We also note that the rate of expansion of the universe during the second inflation is much lower than in the case of the first inflation $H_{s e c} \ll H_{\text {inf }}$, i.e., one has the condition $\frac{\mu_{1}}{c} \gg \mu_{3}$.

Therefore, the models under consideration imply the exit from the first inflationary accelerated expansion stage (for $\mu_{2}= \pm 1$ we have the dynamics corresponding to the radiation domination stage) and the second accelerated expansion of the universe as well. Thus, these dynamics can be considered as the combination of de Sitter and Friedmann solutions and corresponds to the correct change in the stages of the universe's expansion.

\section{The Exact and Approximate Solutions for EPL Inflation from Conformal Connection with $f(R)$-Gravity}

To make a comparison of the scalar field gravity (1) with $f(R)$-gravity with the action $[1,39,40]$

$$
S=\int d^{4} x \sqrt{-g}[f(R)]
$$

we will use the following relations [40]

$$
\begin{aligned}
& R=\left[\sqrt{6} \frac{d V}{d \phi}+4 V\right] \exp \left(\sqrt{\frac{2}{3}} \phi\right), \\
& f=\left[\sqrt{6} \frac{d V}{d \phi}+2 V\right] \exp \left(2 \sqrt{\frac{2}{3}} \phi\right),
\end{aligned}
$$

which connect $f(R)$-gravity and models based on Einstein gravity in parametric form.

Thus, one can use the relation (30) and (31) to put in accordance cosmological models based on Einstein gravity and $f(R)$-gravity on the basis of the exact (6) and approximate (8) expressions for the potential of a scalar field. In paper [41] the exact relations between potentials in Einstein frame and the type of $f(R)$-gravity were reconstructed by the superpotential method.

Looking for connection of the potential (15) with Starobinsky potential [1] we set $\mu_{2}=\sqrt{2 / 3}$. Then, from the Equations (30) and (31) we derive

$$
f(R)=\frac{\mu_{1}}{\mu_{3}} R+\frac{1}{24 \mu_{3}^{2}} R^{2} .
$$


Further, choosing the model's parameters for solutions (10)-(15) as follows:

$$
\begin{aligned}
& -\mu_{1}=\mu_{3}= \pm \frac{1}{2} m, \text { for } n=0, \pm 2, \pm 4, \ldots \\
& \mu_{1}=\mu_{3}= \pm \frac{1}{2} m, \text { for } n= \pm 1, \pm 3, \pm 5, \ldots
\end{aligned}
$$

from expression (15), we get the potential

$$
V_{S R}(\phi)=\frac{3}{4} m^{2}\left(1-e^{-\sqrt{\frac{2}{3}} \phi}\right)^{2},
$$

which exactly corresponds to the Starobinsky potential $[1,40,65,66]$ and non-minimal Higgs potential $[67,68]$ as well. The parameter $m=1.13 \times 10^{-5}$ [71] can be considered as the mass of the scalar field. Also, we note, that this potential leads to the particular case of the two-parametric geometric modified gravity model introduced in Starobinsky's paper [1]

$$
f(R)=R+\frac{1}{6 m^{2}} R^{2},
$$

which generalised Einstein gravity by the second quadratic term in curvature.

The Starobinsky gravity model as a special case of $f(R)$ gravity was considered in astrophysics and cosmology (see, for example, [72-74], and literature cited therein). Also the relation of the Starobinsky model to modified gravity and supergravity is discussed in [75-79].

Also, we note that, for the choice:

$$
\begin{array}{r}
\mu_{1}=\mu_{3}= \pm \frac{1}{2} m, \text { for } n=0, \pm 2, \pm 4, \ldots \\
-\mu_{1}=\mu_{3}= \pm \frac{1}{2} m, \text { for } n= \pm 1, \pm 3, \pm 5, \ldots
\end{array}
$$

one has the following expressions for the potential and the type of $f(R)$-gravity

$$
\begin{aligned}
& V_{S R}(\phi)=\frac{3}{4} m^{2}\left(1+e^{-\sqrt{\frac{2}{3}} \phi}\right)^{2}, \\
& f(R)=-R+\frac{1}{6 m^{2}} R^{2},
\end{aligned}
$$

with negative scalar curvature, which do not correspond to the case of the Starobinsky inflation. For the following analysis in this section, we will only consider solutions (35) and (36) with parameters (33) and (34).

Taking into account the expression for the Hubble parameter (28), which implies the positive values of the constant $\mu_{3}>0$, after substituting constants (33) with upper sign into solutions (12)-(14) one has

$$
\begin{aligned}
& \phi(t)=\sqrt{\frac{3}{2} \ln \left(-\frac{2}{3} m t+c\right)+n \frac{i \pi}{\mu_{2}},} \\
& H(t)=\frac{3 m}{2(2 m t-3 c)}+\frac{1}{2} m, \\
& a(t)=a_{0} \exp \left(\frac{1}{2} m t\right)(-2 m t+3 c)^{3 / 4} .
\end{aligned}
$$

As one can see, solutions (41)-(43) imply a singularity at time $t_{*}=3 c / 2 m$, which cannot be eliminated by choosing the constant $c$. Thus, we will not consider these solutions further, since the phenomenologically correct cosmological solutions should be valid for an arbitrarily cosmic time. 
For the case of substituting constants (34) with upper sign into solutions (12)-(14), for the positive constant $c$, one has non-singular solutions

$$
\begin{aligned}
& \phi(t)=\sqrt{\frac{3}{2}} \ln \left(\frac{2}{3} m t+c\right)+n \frac{i \pi}{\mu_{2}}, \\
& H(t)=\frac{3 m}{2(2 m t+3 c)}+\frac{1}{2} m, \\
& a(t)=a_{0} \exp \left(\frac{1}{2} m t\right)(2 m t+3 c)^{3 / 4},
\end{aligned}
$$

which correspond to the partial case of the cosmological solutions for EPL inflation (10)-(15) corresponding to the Starobinsky model for the case of the slow-roll approximation.

For the potential (11), corresponding to exact solutions, with $\mu_{2}=\sqrt{2 / 3}$ from the Equations (30) and (31) we obtain

$$
f(R)=\frac{\mu_{1}}{\mu_{3}} R+\frac{1}{24 \mu_{3}^{2}} R^{2}+\frac{8}{3} \mu_{1}^{2}
$$

If we additionally chose $\mu_{1}=0$ we the get de Sitter solution

$$
V(\phi)=3 \mu_{3}^{2}, \quad \phi(t)=\sqrt{\frac{3}{2}} \ln (c)+n \frac{i \pi}{\mu_{2}}, \quad H(t)=\mu_{3}, \quad a(t)=a_{0} \exp \left(\mu_{3} t\right),
$$

which correspond to the quadratic term only

$$
f_{d S}(R)=\frac{1}{24 \mu_{3}^{2}} R^{2}
$$

Therefore, the quadratic correction in curvature $R^{2}$ determines the accelerated expansion of the universe in the Starobinsky inflationary model, which is correct in the case of the slow-roll approximation analysis as well.

For the case

$$
\mu_{1}=\mu_{3}=\frac{1}{2} m, \text { for } n= \pm 1, \pm 3, \pm 5, \ldots
$$

from (47) and (15) we have the Starobinsky gravity model with the additional cosmological constant

$$
f(R)=R+\frac{1}{6 m^{2}} R^{2}+\frac{2}{3} m^{2}
$$

and the following potential in Einstein frame

$$
V(\phi)=\frac{27}{20} m^{2}\left(1-\frac{5}{9} e^{-\sqrt{\frac{2}{3}} \phi}\right)^{2}-\frac{3}{5} m^{2}
$$

with the same corresponding expressions for evolution of the scalar field and universe expansion (44)-(46) as in the case of the slow-roll approximation.

Thus, the Starobinsky inflation can be considered as the partial case of exponential power-law inflation with the specific choice of the parameters $\mu_{1}, \mu_{2}, \mu_{3}$ for the complex scalar field with the variable real part and constant imaginary part. For the constant parameter $\mu_{2} \neq \sqrt{2 / 3}$ one does not have the explicit expression for the type of $f(R)$-gravity from Equations (30) and (31). However, one can consider EPL inflation in the Einstein frame, which is connected with $f(R)$-gravity expressed in parametric form.

Also, in the general case, the solutions for the canonical scalar field, i.e., for the constant $n=0$ in expression (12) can be considered as the correct cosmological solutions with dynamics defined by expressions (13) and (14) and corresponding potential (11) with an upper positive sign. 


\section{The Exponential Power-Law Inflation Based on Chiral Cosmological Models}

Chiral cosmological models (CCM) are widely used both to describe the inflationary stage of the early universe and to describe the stage of the second accelerated expansion [29-34]. We also note that on the basis of conformal transformations of the metric of space-time, it is possible to define a correspondence between CCM and cosmological models based on modified theories of gravity [80-83].

The action for the Chiral Cosmological Models (CCM) with $K$ scalar fields $\phi^{A}\left(\bar{\phi}=\phi^{1}, \phi^{2}, \ldots, \phi^{K}\right)$ is

$$
S=\int d^{4} x \sqrt{-g}\left[\frac{1}{2} R-\frac{1}{2} h_{A B} \partial_{\mu} \phi^{A} \partial_{\nu} \phi^{B} g^{\mu \nu}-V(\tilde{\phi})\right],
$$

where $h_{A B}=h_{A B}\left(\phi^{E}\right)$ is the metric tensor of a target (fields) space, and indexes $A, B, E=1, \ldots, \mathrm{K}$.

For the CCM with $K$-fields, in the spatially flat Friedmann-Robertson-Walker metric, from the action (53) one has the following dynamic equations $[29,30]$

$$
\begin{aligned}
& 3 H^{2}=\frac{1}{2} h_{A B} \dot{\phi}^{A} \dot{\phi}^{B}+V(\bar{\phi}), \\
& -\dot{H}=\frac{1}{2} h_{A B} \dot{\phi}^{A} \dot{\phi}^{B}, \\
& h_{E B}\left(\ddot{\phi}^{B}+3 H \dot{\phi}^{B}\right)+V,_{E}=0 .
\end{aligned}
$$

In paper [30] it was shown that this system of similar scalar fields $\phi^{A}$ can be reduced to the equations

$$
\begin{aligned}
& V(\bar{\phi})=V(\varphi)=3 H^{2}+\dot{H}, \\
& \dot{\varphi}^{2}=-2 \dot{H},
\end{aligned}
$$

for a single effective field $\varphi$ which is connected with CCM-fields $\phi^{A}$ as

$$
\varphi(t)= \pm \frac{\sqrt{n}}{2} \sum_{A=1}^{K} \phi^{A}(t), \quad \phi^{A}(t)= \pm \frac{2}{K \sqrt{n}} \varphi(t),
$$

under the following connections between diagonal and non-diagonal components of the metric tensor of target space

$$
\begin{aligned}
& \sum_{B}^{K} h_{E B}=\frac{n}{K} \text { for all } E, \\
& h_{E B}=h_{B E} \quad \text { for } E \neq B,
\end{aligned}
$$

where $n$ is an arbitrary constant.

One can write the condition (60) in explicit form, which corresponds to the following connection between diagonal components and non-diagonal ones of the metric of target (fields) space

$$
h_{11}+h_{12}+\ldots+h_{1 K}=\ldots=h_{K 1}+h_{K 2}+\ldots+h_{K K}=\frac{n}{K} .
$$

Therefore, one can use solutions (57) and (58) for one effective field $\varphi$ to generate the exact solutions for multi-field chiral cosmological models under conditions (60) and (61). Thus, the target spaces with the conditions (60) and (61) on the components of their metric can be called as reducing target spaces. 
In the particular case of two scalar fields $\phi$ and $\psi$ (for $K=2$ ), the dynamics Equations (54)-(56) can be written as follows $[29,30]$

$$
\begin{gathered}
3 H^{2}=\frac{1}{2} h_{11} \dot{\phi}^{2}+h_{12} \dot{\phi} \dot{\psi}+\frac{1}{2} h_{22} \dot{\psi}^{2}+V(\phi, \psi), \\
-\dot{H}=\frac{1}{2} h_{11} \dot{\phi}^{2}+h_{12} \dot{\phi} \dot{\psi}+\frac{1}{2} h_{22} \dot{\psi}^{2}, \\
3 H\left(h_{11} \dot{\phi}+h_{12} \dot{\psi}\right)+\frac{\partial}{\partial t}\left(h_{11} \dot{\phi}+h_{12} \dot{\psi}\right)-\frac{1}{2} \frac{\partial h_{11}}{\partial \phi} \dot{\phi}^{2}-\frac{\partial h_{12}}{\partial \phi} \dot{\phi} \dot{\psi}-\frac{1}{2} \frac{\partial h_{22}}{\partial \phi} \dot{\psi}^{2}+\frac{\partial V}{\partial \phi}=0, \\
3 H\left(h_{12} \dot{\phi}+h_{22} \dot{\psi}\right)+\frac{\partial}{\partial t}\left(h_{12} \dot{\phi}+h_{22} \dot{\psi}\right)-\frac{1}{2} \frac{\partial h_{11}}{\partial \psi} \dot{\phi}^{2}-\frac{\partial h_{12}}{\partial \psi} \dot{\phi} \dot{\psi}-\frac{1}{2} \frac{\partial h_{22}}{\partial \psi} \dot{\psi}^{2}+\frac{\partial V}{\partial \psi}=0 .
\end{gathered}
$$

In reducing target space with components (60) and (61) one has the following exact solutions of Equations (63)-(66) for EPL inflation with canonical scalar field

$$
\begin{gathered}
V(\phi)=\mu_{1}^{2}\left(3-2 \mu_{2}^{2}\right) \exp \left[-\mu_{2} \sqrt{n}(\phi+\psi)\right]+6 \mu_{1} \mu_{3} \exp \left[-\frac{\mu_{2} \sqrt{n}}{2}(\phi+\psi)\right]+3 \mu_{3}^{2}, \\
\phi(t)=\psi(t)= \pm \frac{1}{\mu_{2} \sqrt{n}} \ln \left(2 \mu_{1} \mu_{2}^{2} t+c\right) .
\end{gathered}
$$

Similarly, one can reconstruct the other exact solutions for CCM with a reducing target space based on solutions for single field cosmological models.

An important difference between inflation with multiple scalar fields and inflation with one field is the appearance of additional non-adiabatic perturbations and the evolution of cosmological perturbations after crossing the event horizon as a consequence of the interaction between adiabatic perturbations, which can have a significant effect on the values of the spectral parameters of cosmological perturbations.

Taking into account the interaction of perturbations leads to nonlinear effects, since the perturbations of the scalar fields interact in this case, in contrast to the case of models with one scalar field in the linear order of the theory of cosmological perturbations, where all modes develop independently [84-88].

In the general case, $\phi \neq \psi$, at the stage of cosmological inflation, perturbations of the scalar fields $\delta \phi$ and $\delta \psi$, under the conditions $\epsilon=-\frac{\dot{H}}{H^{2}} \ll 1$ and $\delta=-\frac{\ddot{H}}{2 H \dot{H}} \ll 1$ for the slow-roll parameters, one has the following curvature perturbations [87]

$$
\mathcal{R} \simeq H\left(\frac{\delta \phi}{\dot{\phi}}+\frac{\delta \psi}{\dot{\psi}}\right)
$$

and entropy perturbations

$$
S=H\left(\frac{\delta \phi}{\dot{\phi}}-\frac{\delta \psi}{\dot{\psi}}\right) .
$$

For the similar scalar fields $\phi=\psi$ entropy perturbations equal to zero $S=0$, and curvature perturbations $\mathcal{R} \propto H \frac{\delta \phi}{\dot{\phi}}$ correspond to models with one scalar field. Thus, the spectral parameters of cosmological perturbations in the considered chiral cosmological models are calculated similarly to the case of models of the early universe with one scalar field.

The influence of these effects on the deviation of the resulting disturbance spectrum from Gaussian is estimated using the nonlinearity parameter $f_{N L}$ [89]. According to the latest observational data from the PLANCK satellite, the value of the nonlinearity parameter is estimated as $f_{N L}=2.5 \pm 5.7[90,91]$. 
Now let us define the parameter $f_{N L}$, which determines the deviation of the spectrum of cosmological perturbations from the Gaussian, for two-field models as follows

$$
\frac{6}{5} f_{N L}=2 \frac{\frac{u_{H}^{2}}{\sigma_{*}^{\phi}}\left(1-\frac{\gamma_{*}^{\phi}}{\sigma_{*}^{\phi}} u_{H}\right)+\frac{v_{H}^{2}}{\sigma_{*}^{\psi}}\left(1-\frac{\gamma_{*}^{\psi}}{\sigma_{*}^{\psi}} v_{H}\right)+2\left(\frac{u_{H}}{\sigma_{*}^{\phi}}-\frac{v_{H}}{\sigma_{*}^{\psi}}\right)^{2} \mathcal{A}_{H}}{\left(\frac{u_{H}^{2}}{\sigma_{*}^{\phi}}+\frac{v_{H}^{2}}{\sigma_{*}^{\psi}}\right)^{2}},
$$

where

$$
\begin{gathered}
\sigma^{\phi}=\left(\frac{H_{, \phi}^{(1)}}{H}\right)^{2}, \sigma^{\psi}=\left(\frac{H_{, \psi}^{(2)}}{H}\right)^{2}, \quad \gamma^{\phi}=\frac{H_{, \phi \phi}^{(1)}}{H}, \quad \gamma^{\psi}=\frac{H_{, \psi \psi}^{(2)}}{H}, \\
H(\phi, \psi)=H^{(1)}(\phi)+H^{(2)}(\psi), \quad \sigma=\sigma^{\phi}+\sigma^{\psi}=\frac{1}{2} \epsilon, \\
u_{H} \equiv \frac{H_{*}^{(1)}+Z_{e}}{H_{*}}, \quad v_{H} \equiv \frac{H_{*}^{(2)}-Z_{e}}{H_{*}}, \quad Z_{e}=\left(H_{e}^{(2)} \sigma_{e}^{\phi}-H_{e}^{(1)} \sigma_{e}^{\psi}\right) / \sigma_{e}, \\
\mathcal{A}_{H}=-\frac{H_{e}^{2}}{H_{*}^{2}} \frac{\sigma_{e}^{\phi} \sigma_{e}^{\psi}}{\sigma_{e}}\left(\frac{1}{2}-\frac{\gamma_{e}^{s S}}{\sigma_{e}}\right), \\
\gamma^{s S}=\left(\sigma^{\psi} \gamma^{\phi}+\sigma^{\phi} \gamma^{\psi}\right) / \sigma,
\end{gathered}
$$

the indices $(*)$ and $(e)$ denote the crossing of the event horizon and the end of inflation, respectively.

For the case or $\phi=\psi$ and $V(p h i)=V(\psi)$, the parameters of cosmological perturbations at the horizon crossing and at the end of inflation are equal, also one has

$$
u_{H}=v_{H}=1, \quad \sigma^{\phi}=\sigma^{\psi}=\frac{1}{2} \epsilon, \quad \gamma^{\phi}=\gamma^{\psi}=\delta, \quad \gamma^{s s}=\delta, \quad Z_{e}=0 .
$$

Thus, the nonlinearity parameter is

$$
\frac{6}{5} f_{N L}=\epsilon-\delta \ll 1,
$$

hence, the spectrum of scalar perturbations for the considered class of models can be considered Gaussian, as in the case of models with one scalar field.

The proposed approach simplifies the initially more complex cosmological models and, in this case, only special classes of solutions are considered, associated with the original model of the early universe with one canonical scalar field in the context of its generalisation.

\section{The Exponential Power-Law Inflation in GR-Like Cosmological Models Based on Generalised Scalar-Tensor Gravity}

Scalar-tensor gravity (STG) with non-minimal coupling of a scalar field to curvature are important extensions of GR explaining the initial inflationary evolution, as well as the late accelerating expansion of the universe [48-52]. For the very early universe approaching the Planck scale one can consider Einstein gravity with some corrections as the effective theory of the quantum gravity. The effective supergravity action from superstrings induces correction terms of higher order in the curvature, which may play a significant role in the early universe.

Now, we consider generalised scalar-tensor gravity (GSTG) theory with the action [50,51,92-94]

$$
S=\frac{1}{2} \int d^{4} x \sqrt{-g}\left[F(\phi, R)-\omega(\phi) g^{\mu v} \partial_{\mu} \phi \partial_{\nu} \phi-2 V(\phi)\right],
$$


where

$$
F(\phi, R)=R+f(\phi) R+\xi(\phi) R_{G B}^{2}
$$

where $f(\phi)$ defines a non-minimal coupling of a scalar field with Ricci scalar and $\xi(\phi)$ defines a non-minimal coupling of a scalar field with Gauss-Bonnet scalar $R_{G B}^{2}=R_{\mu v \rho \sigma} R^{\mu v \rho \sigma}-4 R_{\mu v} R^{\mu v}+R^{2}$.

The equations of cosmological dynamics at the stage of inflation in a spatially flat $4 \mathrm{D}$ Friedmann universe can be written as

$$
\begin{aligned}
& E_{1} \equiv 3(1+f) H^{2}+3 H \dot{f}-\frac{\omega}{2} \dot{\phi}^{2}-V(\phi)-12 H^{3} \dot{\xi}=0, \\
& E_{2} \equiv(1+f)\left(3 H^{2}+2 \dot{H}\right)+2 H \dot{f}+\ddot{f}+\frac{\omega}{2} \dot{\phi}^{2}-V(\phi)-8 H^{3} \dot{\xi}-8 H \dot{H} \dot{\xi}-4 H^{2} \ddot{\xi}=0, \\
& E_{3} \equiv \omega \ddot{\phi}+3 \omega H \dot{\phi}+\frac{1}{2} \dot{\phi}^{2} \omega_{\phi}^{\prime}+V_{\phi}^{\prime}-6 H^{2} f_{\phi}^{\prime}-3 \dot{H} F_{\phi}^{\prime}+12 H^{4} \tilde{\zeta}_{\phi}^{\prime}+12 H^{2} \dot{H} \xi_{\phi}^{\prime}=0,
\end{aligned}
$$

with the additional condition

$$
\dot{\phi} E_{3}+\dot{E}_{1}+3 H\left(E_{1}-E_{2}\right)=0 .
$$

Taking into account (84) we conclude that two equations from (81)-(83) are independent only. One can represent the cosmological dynamic equations as

$$
\begin{gathered}
V(\phi)=3(1+f) H^{2}+(1+f) \dot{H}+\frac{5}{2} H \dot{f}+\frac{1}{2} \ddot{f}-10 H^{3} \dot{\xi}-2 H^{2} \ddot{\xi}-4 H \dot{H} \dot{\xi}, \\
\omega(\phi) \dot{\phi}^{2}=H \dot{f}-2(1+f) \dot{H}-\ddot{f}-4 H^{3} \dot{\xi}+8 H \dot{H} \dot{\xi}+4 H^{2} \ddot{\xi} .
\end{gathered}
$$

As one can see, the constant coupling of the scalar field and the Gauss-Bonnet scalar $\xi=$ const does not change the equations of cosmological dynamics for scalar-tensor gravity. The case of GR corresponds to the choice $f=0, \omega=1$ and $\xi=0$.

To characterize the difference between cosmological inflation based on Einstein gravity and generalised scalar-tensor gravity, we introduce the deviation parameters $\Delta_{S T}=\Delta_{S T}(t)$ and $\Delta_{G B}=$ $\Delta_{G B}(t)$, which are connected to the coupling functions as [92]

$$
\begin{aligned}
f(\phi) & =-\Delta_{S T}, \\
\dot{\xi} & =-\frac{\Delta_{G B}}{2 H^{2}} .
\end{aligned}
$$

In terms of these parameters one has the following background dynamic equations

$$
\begin{aligned}
& V(\phi)=3\left(1-\Delta_{S T}\right) H^{2}+\left(1-\Delta_{S T}\right) \dot{H}-\frac{5}{2} H \dot{\Delta}_{S T}-\frac{1}{2} \ddot{\Delta}_{S T}+\dot{\Delta}_{G B}+5 H \Delta_{G B}, \\
& \omega(\phi) \dot{\phi}^{2}=-2\left(1-\Delta_{S T}\right) \dot{H}-H \dot{\Delta}_{S T}+\ddot{\Delta}_{S T}-2 \dot{\Delta}_{G B}+2 H \Delta_{G B} .
\end{aligned}
$$

Now, we define the kinetic function and the deviation parameters as follows [92]

$$
\begin{gathered}
\omega(\phi)=1+\frac{3}{\epsilon}\left(\Delta_{S T}+2 \frac{\Delta_{G B}}{H}\right), \\
\Delta_{S T}(t)=\beta_{S T} a^{-2}(t), \\
\Delta_{G B}(t)=\alpha_{G B} a^{-5}(t),
\end{gathered}
$$

where $\beta_{S T}$ and $\alpha_{G B}$ are the coupling constants of the scalar field with the Ricci scalar and Gauss-Bonnet scalar, respectively. 
After substituting the functions (91)-(93) into the background dynamics Equations (89) and (90) we have the equations

$$
\begin{aligned}
& V(\phi)=3 H^{2}+\dot{H} \\
& \dot{\phi}^{2}=-2 \dot{H} .
\end{aligned}
$$

Further, from (87) and (88) we derive the expressions for non-minimal coupling functions and the kinetic function

$$
\begin{aligned}
& f(t)=-\frac{\beta_{S T}}{a^{2}(t)}, \\
& \dot{\xi}=-\frac{\alpha_{G B}}{2 a^{5} H^{2}}, \\
& \omega(t)=1+\frac{3}{\epsilon a^{2}}\left(\beta_{S T}+\frac{2 \alpha_{G B}}{H a^{3}}\right) .
\end{aligned}
$$

Therefore, for each exact solution of the system (94) and (95) one can find corresponding functions (96)-(98), which characterise the type of GSTG model.

Now, we represent the Equations (94) and (95) and the functions (96)-(98) in terms of a scalar field $\phi$ as the argument on the basis of the following relations

$$
\begin{aligned}
& \dot{H}=-2 H_{\phi}^{\prime 2}, \\
& \dot{\xi}=\xi_{\phi}^{\prime} \dot{\phi}=-2 \tilde{\xi}_{\phi}^{\prime} H_{\phi}^{\prime} \\
& a(\phi)=a_{0} \exp \left(-\frac{1}{2} \int \frac{H}{H_{\phi}^{\prime}} d \phi\right) .
\end{aligned}
$$

As the result, Equations (94) and (95) are transformed to the Ivanov-Salopek-Bond equations for GR $[9,10]$

$$
\begin{aligned}
& V(\phi)=3 H^{2}-2 H_{\phi}^{\prime 2} \\
& \dot{\phi}=-2 H_{\phi}^{\prime}
\end{aligned}
$$

with corresponding non-minimal coupling and kinetic functions

$$
\begin{aligned}
& f(\phi)=-\frac{\beta_{S T}}{a_{0}^{2}} \exp \left(\int \frac{H}{H_{\phi}^{\prime}} d \phi\right), \\
& \xi_{\phi}^{\prime}=\frac{\alpha_{G B}}{4 a_{0}^{5} H_{\phi}^{\prime} H^{2}} \exp \left(\frac{5}{2} \int \frac{H}{H_{\phi}^{\prime}} d \phi\right), \\
& \omega(\phi)=1+\frac{3}{2}\left(\frac{H}{H_{\phi}^{\prime}}\right)^{2} \exp \left(\int \frac{H}{H_{\phi}^{\prime}} d \phi\right)\left[\beta_{S T}+\frac{2 \alpha_{G B}}{H} \exp \left(\frac{3}{2} \int \frac{H}{H_{\phi}^{\prime}} d \phi\right)\right] .
\end{aligned}
$$

For EPL with a Hubble parameter (10), in the general case, non-minimal coupling function $\xi=\xi(\phi)$ can be found in quadratures only, however, for $\mu_{2}=\sqrt{5 / 4}, \sqrt{5 / 6}$ it is possible to obtain this function in explicit form [92].

Further, we consider the evolution of the deviation parameters in terms of the $e$-folds numbers $N=\ln \left(a / a_{0}\right)=-(1 / 2) \int\left(H / H^{\prime}\right) d \phi$. From the expressions (92) and (93) we obtain $\Delta_{S T} \propto \exp (-2 N)$ and $\Delta_{G B} \propto \exp (-5 N)$. Thus, the initial deviations between GR and GST gravity rapidly decrease with 
the expansion of the universe in these models. The value of the $e$-folds numbers at the end of inflation is estimated as $N=50-60$ and, therefore, we have [92]

$$
\begin{aligned}
& \frac{\Delta_{S T}(N=60)}{\Delta_{S T}(N=0)}=e^{-120} \approx 7.7 \times 10^{-53,} \\
& \frac{\Delta_{G B}(N=60)}{\Delta_{G B}(N=0)}=e^{-300} \approx 5.2 \times 10^{-131},
\end{aligned}
$$

where $\Delta_{S T}(N=0), \Delta_{G B}(N=0)$ and $\Delta_{G B}(N=60), \Delta_{G B}(N=60)$ correspond to the values of the deviation parameters at the beginning and at the end of inflation. Thus, for the case $\Delta_{S T}(N=0) \ll e^{120}$ and $\Delta_{G B}(N=0) \ll e^{300}$ at the end of inflation one has $\Delta_{S T} \simeq 0$ and $\Delta_{G B} \simeq 0$.

Also, the parameters of cosmological perturbations for the case of GST gravity under the conditions (91)-(93) coincide with ones in general relativity with high accuracy. Therefore, the proposed approach leads to the generalisation of the inflationary models based on Einstein gravity into the same ones in generalised scalar-tensor gravity.

\section{The Correspondence to the Observational Constraints}

One of the main methods of verification of cosmological models is the comparison of the obtained parameters of cosmological perturbations with observational constraints, which are based on measurements of CMB anisotropy.

These constraints from the PLANCK observations are at the moment estimated as [91]

$$
\begin{aligned}
& \mathcal{P}_{S}=2.1 \times 10^{-9} \\
& n_{S}=0.9663 \pm 0.0041 \\
& r<0.064 \quad \text { (PLANCK 2018/BICEP2/Keck-Array) }
\end{aligned}
$$

The parameters of the cosmological perturbations for Starobinsky and non-minimal Higgs inflation were calculated, for example, in the paper [71]. The spectral index of scalar perturbations $n_{S}$ and tensor-to-scalar ratio $r$ for this type of inflation are $n_{S}=0.967$ and $r=0.003$.

For the exponential power-law inflation with arbitrary constants $\mu_{1}, \mu_{2}, \mu_{3}$ and $c$ these parameters were calculated in the paper [92] on the basis of the exact solutions of background dynamic equations. The resulting relation between the spectral index of scalar perturbations and tensor-to-scalar ratio was determined as

$$
r=\frac{4 s}{n_{S}-3}\left[n_{S}-1+\frac{\mu_{2}\left(\sqrt{2} \mu_{2}-\sqrt{2 \mu_{2}^{2}+4 n_{S}^{2}-16 n_{S}+12}\right)}{\sqrt{2}\left(n_{S}-3\right)}\right],
$$

where the constant parameter $s$ characterizes the normalisation of the amplitude of the tensor perturbations. We will consider the value of this parameter as $s=1$. The value of the power spectrum of the scalar perturbations on the crossing of the Hubble radius $\mathcal{P}_{S}=\mathcal{A}_{S}^{2}=2.1 \times 10^{-9} \mathrm{can}$ be always obtained by the choice of the constants $\mu_{1}, \mu_{3}$ and $c$ for any $\mu_{2}$ [92].

For the case of the Harrison-Zeldovich spectrum $\left(n_{S}=1\right)$ the expression (112) gives $r=0$, i.e., the absence of relic gravitational waves (tensor perturbations) for any value of $\mu_{2}$. Also, for $\mu_{2} \rightarrow \infty$ one has $r=0$ for any value of $n_{S}$ as well.

For Starobinsky and non-minimal Higgs inflation with $\mu_{2}=\sqrt{2 / 3}$ and $n_{S}=0.967$ from the relation (112) one has $r=0.003$, which corresponds to the result obtained in [71]. Also, one can calculate the parameters of cosmological perturbations for the other values of the constant $\mu_{2}$.

On Figure 1 the dependences of the tensor-to-scalar ratio $r$ on the spectral index of scalar perturbations $n_{S}$ for various values of the parameter $\mu_{2}$, which corresponds to the observational constraints, are shown. 


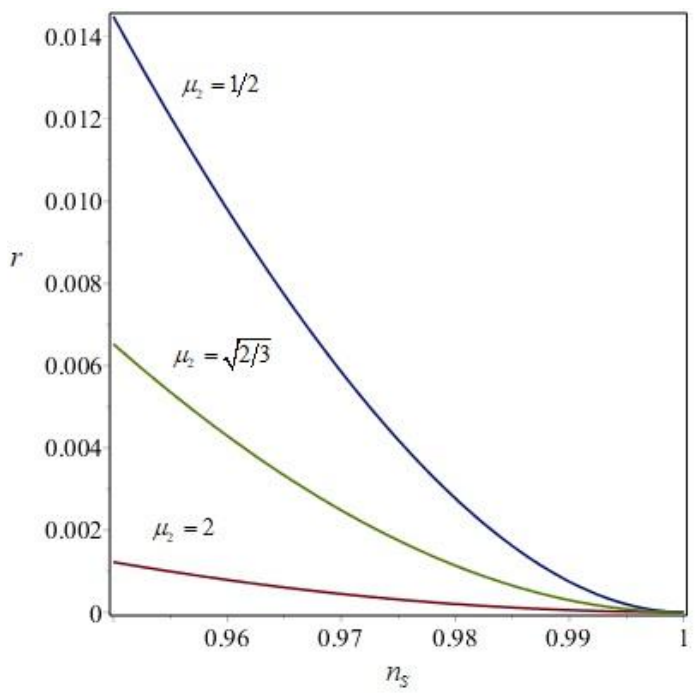

Figure 1. The dependence $r=r\left(n_{S}\right)$ for different values of the constant $\mu_{2}=1 / 2, \sqrt{2 / 3}, 2$.

As one can see, exponential power-law inflation allows to satisfy any restrictions on the value of the tensor-to-scalar ratio by choosing the parameter $\mu_{2}$.

\section{EPL Inflation Based on Scalar-tensor Gravity with Quadratic Connection $H \propto \sqrt{F}$}

Now, we consider the inflationary models based on the action (79) with $F(\phi)=1+f(\phi), \xi(\phi)=0$ and additional relation $H=\lambda \sqrt{F}$, where $\lambda=$ const. The physical content of this relation is that the non-minimal coupling of a scalar field and curvature $F(\phi)=1+\delta F(\phi)$, otherwise the deviations from Einstein gravity, induce the corresponding deviations of dynamics from a pure exponential expansion $H \neq \lambda$ and the deviations of the potential from the constant value $V(\phi)=3 \lambda^{2}+\delta V(\phi)[70,95,96]$.

For the Hubble parameter $H=\lambda+g(t)$, where $g(t)$ is a some function of cosmic time, from Equations (81)-(83) (with $\xi(\phi)=0$ ) one has the system of following dynamic equations $[70,96]$

$$
\begin{gathered}
F(t)=\left(1+\frac{g(t)}{\lambda}\right)^{2}, \\
V(\phi(t))=\frac{1}{\lambda^{2}}\left[3(g(t)+\lambda)^{4}+6(g(t)+\lambda)^{2} \dot{g}+\dot{g}^{2}+(g(t)+\lambda) \ddot{g}\right], \\
\omega(\phi(t)) \dot{\phi}^{2}=-\frac{2}{\lambda^{2}}\left[\dot{g}^{2}+(g(t)+\lambda) \ddot{g}\right] .
\end{gathered}
$$

In the general case, one can obtain the exact cosmological solutions for different dynamics on the basis of Equations (113)-(115) [70,95,96].

For the case of EPL dynamics with $g(t)=m / t$, where $m$ is a some constant, the solutions of Equations (113)-(115) can be written as [70]

$$
\begin{gathered}
V(\phi)=\sum_{k=0}^{4} C_{k}[\Psi(\phi)]^{k}, \\
F(\phi)=\sum_{k=0}^{2} A_{k}[\Psi(\phi)]^{k}, \\
\omega(\phi)=-\frac{2}{U(\phi)} \sum_{k=3}^{4} B_{k}[\Psi(\phi)]^{k}, \quad \dot{\phi}^{2} \equiv U(\phi), \\
t^{-1} \equiv \Psi(\phi),
\end{gathered}
$$


where $\Psi(\phi)$ is some function of a scalar field $\phi$, and the constants $A_{k}, B_{k}, C_{k}$ are

$$
\begin{gathered}
A_{0}=1, A_{1}=\frac{2 m}{\lambda}, A_{2}=\frac{m^{2}}{\lambda^{2}}, \\
B_{3}=\frac{2 m}{\lambda}, B_{4}=\frac{3 m^{2}}{\lambda^{2}}, \\
C_{0}=3 \lambda^{2}, \quad C_{1}=12 m \lambda, \quad C_{2}=6 m(3 m-1), \\
C_{3}=\frac{2 m}{\lambda}\left(6 m^{2}-6 m+1\right), \quad C_{4}=\frac{3 m^{2}}{\lambda^{2}}\left(m^{2}-2 m+1\right) .
\end{gathered}
$$

In the paper [70] the exact solutions for EPL inflation with power-law and exponential potentials were obtained, also the numerical analysis of the solutions for Higgs potential, Higgs-Starobinsky potential, Coleman-Weinberg potential and quadratic potential for a massive scalar field were made.

Also, it was shown, that for parameter $\lambda^{2} \sim 10^{-12}$ such models will satisfy the observational constraints (109)-(111) on the values of cosmological perturbation parameters. The power spectrum of tensor perturbations (relic gravitational waves) on the crossing of the Hubble radius $(k=a H)$ has a constant value [70]

$$
\mathcal{P}_{T}=\frac{2 \lambda^{2}}{\pi^{2}} \approx \frac{2}{\pi^{2}} \times 10^{-12}=\text { const },
$$

the corresponding spectral index $n_{T}=0$, and amplitude of relic gravitational waves $\mathcal{A}_{T}=\mathcal{P}_{T}^{1 / 2} \approx$ $\frac{\sqrt{2}}{\pi} \times 10^{-6}$.

We also note that in the paper [70] it was shown that cosmological models based on the quadratic connection between the Hubble parameter and coupling function $H=\lambda \sqrt{F}$ with exponential power-law dynamics $H(t)=\frac{m}{t}+\lambda$ correspond to observational constrains on the parameter of cosmological perturbations for any potential of a scalar field and any expansion rate of the universe.

\section{Reconstruction of STG Parameters from Physical Potentials}

Due to the fact that the scalar field potential is of key importance for determining physical processes at the stage of cosmological inflation, it is precisely the potential $V(\phi)$ that is specified for constructing models of the early universe. The form of the scalar field potential is determined from elementary particle physics, quantum field theory, theories of unifying fundamental interactions, such as supersymmetric theories and string theories in the context of the inflationary paradigm. We will call the potentials of a scalar field associated with these mechanisms as physical potentials. Physical mechanisms corresponding to a large number of inflationary potentials were considered in the reviews $[97,98]$.

Now, we consider $\Psi(\phi)$ as the following function of some known physical potential

$$
\Psi(\phi)=\left[\frac{V_{p h}(\phi)}{C_{4}}\right]^{1 / 4}=t^{-1}, \quad C_{4} \neq 0, \quad m \neq 1,
$$

where $V_{p h}$ is some physical potential of a scalar field, and we exclude a special case $C_{4}=0$ for $m=1$.

Thus, from (116) we have the following exact expression for the potential

$$
V(\phi)=V_{p h}(\phi)+\frac{C_{3}}{C_{4}^{3 / 4}} V_{p h}^{3 / 4}(\phi)+\frac{C_{2}}{C_{4}^{1 / 2}} V_{p h}^{1 / 2}(\phi)+\frac{C_{1}}{C_{4}^{1 / 4}} V_{p h}^{1 / 4}(\phi)+C_{0},
$$


For the case $\lambda \sim 10^{-6}$ and $m \gg 1$ one has

$$
\begin{aligned}
& \frac{C_{3}}{C_{4}^{3 / 4}}=\frac{m\left(6 m^{2}-6 m+1\right) \lambda^{1 / 2}}{[m(m-1)]^{3 / 2}} \sim 10^{-3}, \\
& \frac{C_{2}}{C_{4}^{1 / 2}}=\frac{6(3 m-1) \lambda}{m-1} \sim 10^{-5}, \\
& \frac{C_{1}}{C_{4}^{1 / 4}}=\frac{12 m \lambda^{3 / 2}}{[m(m-1)]^{1 / 2}} \sim 10^{-8}, \\
& C_{0}=3 \lambda^{2} \sim 10^{-12} .
\end{aligned}
$$

For the coupling function (117) one has following exact expression

$$
F(\phi)=1+\frac{A_{2}}{C_{4}^{1 / 2}} V_{p h}^{1 / 2}(\phi)+\frac{A_{1}}{C_{4}^{1 / 4}} V_{p h}^{1 / 4}(\phi),
$$

for the case $\lambda \sim 10^{-6}$ and $m \gg 1$ we obtain

$$
\begin{aligned}
& \frac{A_{2}}{C_{4}^{1 / 2}}=\frac{m}{(m-1) \lambda} \sim 10^{6}, \\
& \frac{A_{1}}{C_{4}^{1 / 4}}=\frac{2 m}{[\lambda m(m-1)]^{1 / 2}} \sim 10^{3} .
\end{aligned}
$$

The kinetic function (118) can be written as

$$
\omega(\phi)=-\frac{2}{U(\phi)}\left[\frac{B_{4}}{C_{4}} V_{p h}(\phi)+\frac{B_{3}}{C_{4}^{3 / 4}} V_{p h}^{3 / 4}(\phi)\right],
$$

For the case $\lambda \sim 10^{-6}$ and $m \gg 1$ one has

$$
\begin{aligned}
& \frac{B_{4}}{C_{4}}=\frac{3}{(m-1)^{2}} \sim m^{-2}, \\
& \frac{B_{3}}{C_{4}^{3 / 4}}=\frac{2 m \lambda^{1 / 2}}{[m(m-1)]^{3 / 2}} \sim 10^{-3} \times m^{-2} .
\end{aligned}
$$

Therefore, for this case the kinetic energy can be estimated as $X=-\frac{1}{2} \omega(\phi) U(\phi)=-\frac{1}{2} \omega(\phi) \dot{\phi}^{2} \sim$ $m^{-2} V_{p h} \ll V_{p h}$ for $m \gg 1$. This result corresponds to the slow-roll condition for the predominance of the potential energy of the scalar field over the kinetic one.

Now, we consider the procedure of reconstruction of the model's parameters for a priori defined physical potential $V_{p h}(\phi)$. As the first example, we consider the potential for polynomial chaotic inflation $[97,98]$

$$
V_{p h}(\phi)=V_{P}(\phi)=V_{0} \phi^{p},
$$

where $p$ is an arbitrary constant.

From the Equation (121) we obtain the evolution of the scalar field

$$
\phi(t)=(\beta t)^{-4 / p}, \quad \beta=\left(V_{0} / C_{4}\right)^{1 / 4} .
$$

From the expression (134) one has the inverse dependence

$$
t=\frac{1}{\beta} \phi^{-p / 4}
$$


From the relation $U(\phi)=\dot{\phi}^{2}$ we obtain

$$
U(\phi)=\left(\frac{4}{p t}\right)^{2}(\beta t)^{-8 / p}=\left(\frac{4}{p \beta}\right)^{2} \phi^{\frac{p}{2}+2} .
$$

The exact expressions for the potential, coupling and kinetic function is given by the relations (122), (127) and (130).

By a similar way one can obtain the parameters of STG for the other physical potentials:

- Higgs potential

$$
\begin{aligned}
& V_{p h}(\phi)=V_{H}(\phi)=\frac{\lambda_{H}}{4}\left(\phi^{2}-v^{2}\right)^{2}, \\
& \phi(t)= \pm\left(v^{2}+\frac{1}{\beta_{H}^{2} t^{2}}\right)^{1 / 2}, \\
& \beta_{H}=\left(\frac{\lambda_{H}}{4 C_{4}}\right)^{1 / 4}, \\
& U(\phi)=\frac{\beta_{H}^{2}}{\phi^{2}}\left(\phi^{2}-v^{2}\right)^{3},
\end{aligned}
$$

where $\lambda_{H}$ is the Higgs coupling constant and $v$ is the vacuum expectation value of the Higgs field.

- Higgs-Starobinsky potential

$$
\begin{aligned}
& V_{p h}(\phi)=V_{0}\left(1-e^{-\sqrt{\frac{2}{3}} \phi}\right)^{2}, \\
& \phi(t)=-\sqrt{6} \ln \left|1-\frac{1}{\beta_{H S}^{2} t^{2}}\right|, \\
& \beta_{H S}=\left(V_{0} / C_{4}\right)^{1 / 4}, \\
& U(\phi)=6 \beta_{H S}^{2} e^{-\sqrt{\frac{2}{3}} \phi}\left(1-e^{\sqrt{\frac{2}{3}} \phi}\right)^{3} .
\end{aligned}
$$

- Coleman-Weinberg potential

$$
\begin{aligned}
& V_{p h}(\phi)=V_{C W}(\phi)=\alpha \phi^{4}\left(\ln \left(\frac{\phi}{v_{\phi}}\right)-\frac{1}{4}\right)+\frac{\alpha}{4} v_{\phi \prime}^{4}, \\
& \phi(t)=v_{\phi}\left[\frac{1-\left(v_{\phi} \beta_{C W} t\right)^{-4}}{W\left(e^{-1}\left[1-\left(v_{\phi} \beta_{C W} t\right)^{-4}\right]\right)}\right]^{1 / 4}, \\
& \beta_{C W}=\left(\alpha / C_{4}\right)^{1 / 4}, \\
& U(\phi)=\frac{\beta_{C W}^{2}\left(\frac{4}{\alpha} V_{C W}\right)^{5 / 2}}{\left[W\left(\frac{4}{e \alpha} V_{C W}-\frac{v_{\phi}^{4}}{e}\right)+1\right]^{2}}\left[\frac{W\left(\frac{4}{e \alpha} V_{C W}-\frac{v_{\phi}^{4}}{e}\right)}{\frac{4}{\alpha} V_{C W}-v_{\phi}^{4}}\right]^{3 / 2},
\end{aligned}
$$

where $W$ denotes the Lambert function.

Similarly, it is possible to reconstruct the evolution of the scalar field and the parameters of the scalar-tensor gravity theory for other physical potentials.

The exact expression for the potential (122) corresponds to the considered physical potentials with an accuracy of $10^{-3}$ for the case $\lambda \sim 10^{-6}$ and $m \gg 1$. 
Therefore, one can consider the potential of a scalar field as

$$
V(\phi)=V_{p h}(\phi)+\text { small corrections, } V(\phi) \approx V_{p h}(\phi),
$$

after neglecting the small terms of order $\mathcal{O}\left(10^{-3}\right)$ and higher.

We also note that the terms in expressions (127) and (130) differ by three orders as well, therefore, with the same accuracy after neglecting the terms of order $O\left(10^{-3}\right)$, one can write the coupling and kinetic functions in the following form

$$
\begin{gathered}
F(\phi) \approx 1+10^{6} \times V_{p h}^{1 / 2}(\phi), \\
\omega(\phi) \approx-m^{-2} \times\left(\frac{V_{p h}(\phi)}{U(\phi)}\right) .
\end{gathered}
$$

Thus, it is possible to reconstruct the parameters of the scalar-tensor gravity theory corresponding to any potential of a scalar field for inflationary models under consideration.

\section{Discussion}

In this article, we have given a review of the methods for constructing and analysing models of cosmological inflation with exponential-power dynamics based on Einstein gravity and some of its modifications.

Firstly, we considered the exponential power-law inflation on the basis of the exact and approximate cosmological solutions in the framework of General Relativity. The law of evolution of a scalar field, its potential and the nature of the dynamics of the early universe were obtained. Based on the results obtained, it can be argued that the Starobinsky and non-minimal Higgs models are the partial cases of the exponential power-law inflation from a dynamically motivated point of view. On the basis of exact solutions of the equations of cosmological dynamics in the Einstein frame, an expression for Starobinsky $f(R)$-gravity with an additional term corresponding to the cosmological constant

$$
f(R)=R+\frac{1}{6 m^{2}} R^{2}+\frac{2}{3} m^{2},
$$

was obtained. Also, it was shown that the pure exponential expansion of the early universe associated with quadratic correction in curvature $R^{2}$.

Secondly, we used the method of reduction of multifield Chiral Cosmological Models (CCM) to the single field models on the basis of reducing target spaces with components of a metric tensor, which are connected by conditions (60) and (61) for similar scalar fields. This method allows one to generalise the solutions for EPL inflation with one scalar field on this class of CCM. It was also shown that the parameters of cosmological perturbations in these models are calculated similarly to the case of standard inflation models.

Thirdly, we generalise the solutions for EPL inflation on the case of generalised scalar-tensor gravity with non-minimal coupling of a scalar field with the Ricci and Gauss-Bonnet scalars. For such models one can obtain the parameters of these gravity theories from GR cosmological solutions. The parameters of cosmological perturbations in these models are calculated similarly to the case of standard inflation models for such models as well.

An analysis of the observational constraints on the values of the parameters of cosmological perturbations suggests that exponential power-law inflation can satisfy any constraints on the value of the tensor-to-scalar ratio obtained from the observational data.

Further, we considered the other class of cosmological models with quadratic connection between Hubble parameter and function, which define the nature of the coupling of a scalar field and curvature. General Relativistic limit in such models corresponds to de Sitter solutions only. The methods of exact solutions and slow-roll one's construction for such models were considered as well. Also we note that 
a distinctive feature of such models is that they correspond to observational constraints on the values of the parameters of cosmological perturbations for any potential of the scalar field.

Finally, despite the fact that in this paper we considered only the case of exponential power-law inflation, it is obvious that the proposed methods can be used to construct and analyse cosmological solutions for any other dynamics of the early universe.

Author Contributions: Investigation, I.F. and S.C.; writing, I.F. and S.C. All authors have read and agreed to the published version of the manuscript.

Funding: I.F. and S.C. were funded by the Russian Foundation for Basic Research grant No. 20-02-00280 A. I.F. was partly funded by the Russian Foundation for Basic Research grant No. 19-29-11015 mk.

Acknowledgments: S.C. was partially supported by the Russian Government Program of Competitive Growth of Kazan Federal University.

Conflicts of Interest: The authors declare no conflict of interest.

\section{References}

1. Starobinsky, A.A. A New Type of Isotropic Cosmological Models Without Singularity. Phys. Lett. B 1980, 91, 99-102. [CrossRef]

2. Guth, A.H. The Inflationary Universe: A Possible Solution to the Horizon and Flatness Problems. Phys. Rev. D 1981, 23, 139-148. [CrossRef]

3. Linde, A. A New Inflationary Universe Scenario: A Possible Solution of the Horizon, Flatness, Homogeneity, Isotropy and Primordial Monopole Problems. Phys. Lett. B 1982, 108, 389-393. [CrossRef]

4. Linde, A.D. Particle physics and inflationary cosmology. Contemp. Concepts Phys. 1990, 5, 1-362.

5. Zhuravlev, V.M.; Chervon, S.V.; Shchigolev, V.K. New classes of exact solutions in inflationary cosmology. J. Exp. Theor. Phys. 1998, 87, 223-228. [CrossRef]

6. Liddle, A.R.; Parsons, P.; Barrow, J.D. Formalizing the slow roll approximation in inflation. Phys. Rev. D 1994, 50, 7222-7232. [CrossRef] [PubMed]

7. Fomin, I.V. The models of cosmological inflation in the context of kinetic approximation. J. Phys. Conf. Ser. 2016, 731, 012004. [CrossRef]

8. Fomin, I.V. Cosmological inflation models in the kinetic approximation. Theor. Math. Phys. 2017, 191, 781-791. [CrossRef]

9. Ivanov, G.G. Friedmann cosmological models with a nonlinear scalar field. In Gravitation and Theory of Relativity; Kazan University Publishing House: Kazan, Russia, 1981; pp. 54-60.

10. Salopek D.S.; Bond, J.R. Stochastic inflation and nonlinear gravity. Phys. Rev. D 1991, 43, 1005-1031. [CrossRef]

11. Chervon, S.V.; Fomin, I.V.; Barrow, J.D. The method of generating functions in exact scalar field inflationary cosmology. Eur. Phys. J. C 2018, 4, 301. [CrossRef]

12. Fomin, I.V.; Chervon, S.V. Exact and Approximate Solutions in the Friedmann Cosmology. Russ. Phys. J. 2017, 60, 427-440. [CrossRef]

13. Fomin, I.V. Generalized Exact Solutions in the Friedmann Cosmology. Russ. Phys. J. 2018, 61, 843-851. [CrossRef]

14. Fomin, I.V.; Chervon, S.V.; Maharaj, S.D. A new look at the Schrödinger equation in exact scalar field cosmology. Int. J. Geom. Meth. Mod. Phys. 2018, 16, 1950022. [CrossRef]

15. Chervon, S.; Fomin, I.; Yurov V.; Yurov A. Scalar Field Cosmology; World Scientific: Singapore, 2019; p. 263, ISBN 9789811205071.

16. Perlmutter, S.; Supernova Cosmology Project Collaboration. Measurements of $\Omega$ and $\Lambda$ from 42 high redshift supernovae. Astrophys. J. 1999, 517, 565. [CrossRef]

17. Riess, A.G.; Supernova Search Team. Observational evidence from supernovae for an accelerating universe and a cosmological constant. Astrophys. J. 1998, 116, 1009. [CrossRef]

18. Sahni, V.; Starobinsky, A.A. The Case for a positive cosmological Lambda term. Int. J. Mod. Phys. D 2000, 9, 373. [CrossRef]

19. Peebles, P. The Cosmological Constant and Dark Energy. Ev. Mod. Phys. 2003, 75, 559. [CrossRef]

20. Padmanabhan, T. Cosmological constant: The Weight of the vacuum. Phys. Rept. 2003, 380, 235. [CrossRef] 
21. Sahni, V.; Starobinsky, A.A. Reconstructing Dark Energy. Int. J. Mod. Phys. D 2006, 15, 2105. [CrossRef]

22. Rubakov, V.A. Relaxation of the cosmological constant at inflation? Phys. Rev. D 2000, 61, 061501. [CrossRef]

23. Steinhardt, P.J.; Turok, N. Why the cosmological constant is small and positive. Science 2006, 312, 1180. [CrossRef] [PubMed]

24. Tsujikawa, S. Quintessence: A Review. Class. Quant. Grav. 2013, 30, 1180. [CrossRef]

25. Durrive, J.B.; Ooba, J.; Ichiki, K.; Sugiyama, N. Updated observational constraints on quintessence dark energy models. Phys. Rev. D 2018, 97, 043503. [CrossRef]

26. Armendariz-Picon, C.; Mukhanov, V.F.; Steinhardt, P.J. Essentials of k essence. Phys. Rev. D 2001, 63, 103510. [CrossRef]

27. Chiba, T. Tracking K-essence. Phys. Rev. D 2002, 66, 063514. [CrossRef]

28. Sazhin, M.V.; Sazhina, O.S. The scale factor in a Universe with dark energy. Astron. Rep. 2016, 60, 425. [CrossRef]

29. Chervon, S.V. Chiral Cosmological Models: Dark Sector Fields Description. Quant. Matt. 2013, $2,71$. [CrossRef]

30. Chervon, S.V.; Fomin, I.V.; Pozdeeva, E.O.; Sami, M.; Vernov, S.Y. Superpotential method for chiral cosmological models connected with modified gravity. Phys. Rev. D 2019, 100, 063522. [CrossRef]

31. Abbyazov, R.R.; Chervon, S.V. Interaction of chiral fields of the dark sector with cold dark matter. Grav. Cosmol. 2012, 18, 262. [CrossRef]

32. Abbyazov, R.R.; Chervon, S.V.; Muller, V. $\sigma$ CDM coupled to radiation: Dark energy and Universe acceleration. Mod. Phys. Lett. A 2015, 30, 1550114. [CrossRef]

33. Fomin, I.V. The chiral cosmological models with two components. J. Phys. Conf. Ser. 2017, 918, 012009. [CrossRef]

34. Fomin, I.V. Two-Field Cosmological Models with a Second Accelerated Expansion of the Universe. Mosc. Univ. Phys. Bull. 2019, 73, 696. [CrossRef]

35. Starobinsky, A.A. Disappearing cosmological constant in $\mathrm{f}(\mathrm{R})$ gravity. JETP Lett. 2015, 86, 157. [CrossRef]

36. Nojiri, S.; Odintsov, S.D. Modified non-local-F(R) gravity as the key for the inflation and dark energy. Phys. Lett. B 2008, 659, 821. [CrossRef]

37. Clifton, T.; Ferreira, P.G.; Padilla, A.; Skordis, C. Modified Gravity and Cosmology. Phys. Rept. 2012, 513, 1. [CrossRef]

38. Rinaldi, M.; Cognola, G.; Vanzo, L.; Zerbini, S. Reconstructing the inflationary $f(R)$ from observations. JCAP 2014, 1408, 015. [CrossRef]

39. Motohashi, H.; Starobinsky, A.A. $f(R)$ constant-roll inflation. Eur. Phys. J. C 2017, 77, 538. [CrossRef]

40. Aldabergenov, Y.; Ishikawa, R.; Ketov, S.V.; Kruglov, S.I. Beyond Starobinsky inflation. Phys. Rev. D 2018, 98, 083511. [CrossRef]

41. Vernov, S.Y.; Ivanov, V.R.; Pozdeeva, E.O. Superpotential method for F(R) cosmological models. Phys. Part. Nucl. 2020, 51, 744-749. [CrossRef]

42. Nojiri, S.; Odintsov, S.D. Modified gravity with negative and positive powers of the curvature: Unification of the inflation and of the cosmic acceleration. Phys. Rev. D 2003, 68, 123512. [CrossRef]

43. Elizalde, E.; Nojiri, S.; Odintsov, S.D. Late-time cosmology in (phantom) scalar-tensor theory: Dark energy and the cosmic speed-up. Phys. Rev. D 2004, 70, 043539. [CrossRef]

44. Capozziello, S.; De Laurentis, M.; Nojiri, S.; Odintsov, S.D. Evolution of gravitons in accelerating cosmologies: The case of extended gravity. Phys. Rev. D 2017, 95, 083524. [CrossRef]

45. Horndeski, G.W. Second-order scalar-tensor field equations in a four-dimensional space. Int. J. Theor. Phys. 1974, 10, 363. [CrossRef]

46. De Felice, A.; Tsujikawa, S. Inflationary non-Gaussianities in the most general second-order scalar-tensor theories. Phys. Rev. D 2011, 84, 083504. [CrossRef]

47. Starobinsky, A.A.; Sushkov, S.V.; Volkov, M.S. The screening Horndeski cosmologies. J. Cosmol. Astropart. Phys. 2016, 1606, 007. [CrossRef]

48. Fujii, Y.; Maeda, K. The Scalar-tensor Theory of Gravitation; Cambridge University Press: Cambridge, UK, 2003; p. 260. ISBN 9780521037525. [CrossRef]

49. Faraoni, V. Cosmology in Scalar-Tensor Gravity. Fundam. Theor. Phys. 2004, 139, 267. [CrossRef]

50. DeFelice, A.; Tsujikawa, S.; Elliston, J.; Tavakol, R. Chaotic inflation in modified gravitational theories. J. Cosmol. Astropart. Phys. 2011, 1108, 021. [CrossRef] 
51. DeFelice, A.; Tsujikawa, S. Conditions for the cosmological viability of the most general scalar-tensor theories and their applications to extended Galileon dark energy models. J. Cosmol. Astropart. Phys. 2012, 1202, 007. [CrossRef]

52. Fomin, I.; Chervon, S. Inflation with explicit parametric connection between general relativity and scalar-tensor gravity. Mod. Phys. Lett. A 2018, 33, 1850161. [CrossRef]

53. Kanti, P.; Rizos, J.; Tamvakis, K. Singularity free cosmological solutions in quadratic gravity. Phys. Rev. D 1999, 59, 083512. [CrossRef]

54. Guo, Z.K.; Schwarz, D.J. Slow-roll inflation with a Gauss-Bonnet correction. Phys. Rev. D 2010, 81, 123520. [CrossRef]

55. Van de Bruck, C.; Longden, C. Higgs Inflation with a Gauss-Bonnet term in the Jordan Frame. Phys. Rev. D 2016, 93, 063519. [CrossRef]

56. Hikmawan, G.; Soda, J.; Suroso, A.; Zen, F.P. Comment on “Gauss-Bonnet inflation”. Phys. Rev. D 2016, 93, 068301. [CrossRef]

57. Koh, S.; Lee, B.H.; Tumurtushaa, G. Reconstruction of the Scalar Field Potential in Inflationary Models with a Gauss-Bonnet term. Phys. Rev. D 2017, 95, 123509. [CrossRef]

58. Fomin, I.V.; Chervon, S.V. Exact inflation in Einstein-Gauss-Bonnet gravity. Grav. Cosmol. 2017, $23,367$. [CrossRef]

59. Fomin, I.V.; Morozov, A.N. The high-frequency gravitational waves in exact inflationary models with Gauss-Bonnet term. J. Phys. Conf. Ser. 2017, 798, 012088. [CrossRef]

60. Fomin, I.V.; Chervon, S.V. A new approach to exact solutions construction in scalar cosmology with a Gauss-Bonnet term. Mod. Phys. Lett. A 2017, 32, 1750129. [CrossRef]

61. Odintsov, S.D.; Oikonomou, V.K. Viable Inflation in Scalar-Gauss-Bonnet Gravity and Reconstruction from Observational Indices. Phys. Rev. D 2018, 98, 044039. [CrossRef]

62. Pozdeeva, E.O. Generalization of cosmological attractor approach to Einstein-Gauss-Bonnet gravity. Eur. Phys. J. C 2020, 80, 612. [CrossRef]

63. Lovelock, D. The Einstein tensor and its generalizations. J. Math. Phys. 1971, 12, 498. [CrossRef]

64. Ketov, S.V.; Watanabe, N. The f(R) Gravity Function of the Linde Quintessence. Phys. Lett. B 2014, 741, $242-245$. [CrossRef]

65. Whitt, B. Fourth Order Gravity as General Relativity Plus Matter. Phys. Lett. B 1984, 145, 176-178. [CrossRef]

66. Maeda, K.I. Inflation as a Transient Attractor in $R^{2}$ Cosmology. Phys. Rev. D 1988, 37, 858-862. [CrossRef] [PubMed]

67. Bezrukov, F.L.; Shaposhnikov, M. The Standard Model Higgs boson as the inflaton. Phys. Lett. B 2008, 659, 703-706. [CrossRef]

68. Bezrukov, F.L.; Magnin, A.; Shaposhnikov, M. Standard Model Higgs boson mass from inflation. Phys. Lett. B 2009, 675, 88-92. [CrossRef]

69. Gorbunov, D.S.; Panin, A.G. Are $R^{2}$ - and Higgs-inflations really unlikely? Phys. Lett. B 2015, 743, 79-81. [CrossRef]

70. Fomin, I.V.; Chervon, S.V.; Tsyganov, A.V. Generalized scalar-tensor theory of gravity reconstruction from physical potentials of a scalar field. Eur. Phys. J. C 2020, 80, 350. [CrossRef]

71. Mishra, S.S.; Sahni, V.; Toporensky, A.V. Initial conditions for Inflation in an FRW Universe. Phys. Rev. D 2018, 98, 083538. [CrossRef]

72. Arapoglu, S.; Cikintoglu, S.; Eksi, K.Y. Relativistic stars in Starobinsky gravity with the matched asymptotic expansions method. Phys. Rev. D 2017, 96, 084040. [CrossRef]

73. Giudice, G.F.; Lee, H.M. Starobinsky-like inflation from induced gravity. Phys. Lett. B 2014, 733, 58-62. [CrossRef]

74. Capozziello, S.; Nojiri, S.; Odintsov, S.D. The role of energy conditions in $f(R)$ cosmology. Phys. Lett. B 2018, 781, 99. [CrossRef]

75. Channuie, P. Deformed Starobinsky model in gravity's rainbow. Eur. Phys. J. C 2019, 79, 508. [CrossRef]

76. Sebastiani, L.; Cognola, G.; Myrzakulov, R.; Odintsov, S.D.; Zerbini, S. Nearly Starobinsky inflation from modified gravity. Phys. Rev. D 2014, 89, 023518. [CrossRef]

77. Moraes, P.; Sahoo, P.K.; Ribeiro, G.; Correa, R. A Cosmological Scenario from the Starobinsky Model within the $f(R, T)$ Formalism. Adv. Astron. D 2019, 2019, 8574798. [CrossRef] 
78. Odintsov, S.D.; Oikonomou, V.K.; Sebastiani, L. Unification of Constant-roll Inflation and Dark Energy with Logarithmic $R^{2}$-corrected and Exponential $F(R)$ Gravity. Nucl. Phys. B 2017, 923, 608-632. [CrossRef]

79. Ketov, S.V. On the equivalence of Starobinsky and Higgs inflationary models in gravity and supergravity. J. Phys. A 2020, 53, 084001. [CrossRef]

80. Naruko, A.; Yoshida, D.; Mukohyama, S. Gravitational scalar-tensor theory. Class. Quant. Grav. 2016, 33, 09LT01. [CrossRef]

81. Saridakis, E.N.; Tsoukalas, M. Cosmology in new gravitational scalar-tensor theories. Phys. Rev. D 2016, 93, 124032. [CrossRef]

82. Chervon, S.V.; Fomin, I.V.; Mayorova, T.I. Chiral Cosmological Model of $f(R)$ Gravity with a Kinetic Curvature Scalar. Grav. Cosmol. 2019, 25, 205. [CrossRef]

83. Chervon, S.V.; Fomin, I.V.; Mayorova, T.I.; Khapaeva, A.V. Cosmological parameters of f(R) gravity with kinetic scalar curvature. J. Phys. Conf. Ser. 2020, 1557, 012016. [CrossRef]

84. Garcia-Bellido, J.; Wands, D. Metric perturbations in two field inflation. Phys. Rev. D 1996, 53, 5437. [CrossRef] [PubMed]

85. Gordon, C.; Wands, D.; Bassett, B.A.; Maartens, R. Adiabatic and entropy perturbations from inflation. Phys. Rev. D 2000, 63, 023506. [CrossRef]

86. Starobinsky, A.A.; Tsujikawa, S.; Yokoyama, J. Cosmological perturbations from multifield inflation in generalized Einstein theories. Nucl. Phys. B 2001, 610, 383. [CrossRef]

87. Wands, D.; Bartolo, N.; Matarrese, S.; Riotto, A. An Observational test of two-field inflation. Phys. Rev. D 2002, 66, 043520. [CrossRef]

88. Byrnes, C.T.; Choi, K.Y. Review of local non-Gaussianity from multi-field inflation. Adv. Astron. 2010, 2010, 724525. [CrossRef]

89. Bartolo, N.; Komatsu, E.; Matarrese, S.; Riotto, A. Non-Gaussianity from inflation: Theory and observations. Phys. Rept. 2004, 402, 103. [CrossRef]

90. Ade, P. [Planck Collaboration]. Planck 2015 results. XIII. Cosmological parameters. Astron. Astrophys. 2016, 594, A13. [CrossRef]

91. Aghanim, N.; Planck Collaboration. Planck 2018 results. VI. Cosmological parameters. arXiv 2020, arXiv:1807.06209.

92. Fomin, I.V.; Chervon, S.V. Reconstruction of general relativistic cosmological solutions in modified gravity theories. Phys. Rev. D 2019, 100, 023511. [CrossRef]

93. Pozdeeva, E.O.; Sami, M.; Toporensky, A.V.; Vernov, S.Y. Stability analysis of de Sitter solutions in models with the Gauss-Bonnet term. Phys. Rev. D 2019, 100, 083527. [CrossRef]

94. Pozdeeva, E.O.; Gangopadhyay, M.R.; Sami, M.; Toporensky, A.V.; Vernov, S.Y. Inflation with a quartic potential in the framework of Einstein-Gauss-Bonnet gravity. Phys. Rev. D 2020, 102, 043525. [CrossRef]

95. Fomin, I.V.; Chervon, S.V. The exact solutions in verified cosmological models based on generalized scalar-tensor gravity. Eur. Phys. J. C 2018, 78, 918. [CrossRef]

96. Fomin, I.V.; Chervon, S.V. Non-minimal coupling influence on the deviation from de Sitter cosmological expansion. J. Phys. Conf. Ser. 2020, 1557, 012020. [CrossRef]

97. Martin, J.; Ringeval, C.; Vennin, V. Encyclopedia Inflationaris. Phys. Dark Univ. 2014, 5-6, 75. [CrossRef]

98. Grøn, Ø. Predictions of Spectral Parameters by Several Inflationary Universe Models in Light of the Planck Results. Universe 2018, 4, 715. [CrossRef]

Publisher's Note: MDPI stays neutral with regard to jurisdictional claims in published maps and institutional affiliations.

(C) 2020 by the authors. Licensee MDPI, Basel, Switzerland. This article is an open access article distributed under the terms and conditions of the Creative Commons Attribution (CC BY) license (http://creativecommons.org/licenses/by/4.0/). 